ARTICLE

https://doi.org/10.1038/s41467-019-13467-w

\title{
Gut uropathogen abundance is a risk factor for development of bacteriuria and urinary tract infection
}

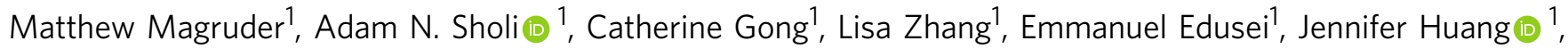
Shady Albakry', Michael J. Satlin², Lars F. Westblade ${ }^{2,3}$, Carl Crawford ${ }^{4}$, Darshana M. Dadhania, ${ }^{1,5}$, Michelle Lubetzky ${ }^{1,5}$, Ying Taur ${ }^{6}$, Eric Littman ${ }^{6}$, Lilan Ling ${ }^{6}$, Philip Burnham7, Iwijn De Vlaminck (1) ${ }^{7}$, Eric Pamer ${ }^{6}$, Manikkam Suthanthiran ${ }^{1,5}$ \& John Richard Lee ${ }^{1,5 *}$

The origin of most bacterial infections in the urinary tract is often presumed to be the gut. Herein, we investigate the relationship between the gut microbiota and future development of bacteriuria and urinary tract infection (UTI). We perform gut microbial profiling using $16 \mathrm{~S}$ rRNA gene deep sequencing on 510 fecal specimens from 168 kidney transplant recipients and metagenomic sequencing on a subset of fecal specimens and urine supernatant specimens. We report that a $1 \%$ relative gut abundance of Escherichia is an independent risk factor for Escherichia bacteriuria and UTI and a 1\% relative gut abundance of Enterococcus is an independent risk factor for Enterococcus bacteriuria. Strain analysis establishes a close strain level alignment between species found in the gut and in the urine in the same subjects. Our results support a gut microbiota-UTI axis, suggesting that modulating the gut microbiota may be a potential novel strategy to prevent UTIs.

\footnotetext{
${ }^{1}$ Division of Nephrology and Hypertension, Department of Medicine, Weill Cornell Medicine, New York, NY, USA. ${ }^{2}$ Division of Infectious Diseases, Department of Medicine, Weill Cornell Medicine, New York, NY, USA. ${ }^{3}$ Department of Pathology and Laboratory Medicine, Weill Cornell Medicine, New York, NY, USA. ${ }^{4}$ Division of Gastroenterology, Department of Medicine, Weill Cornell Medicine, New York, NY, USA. ${ }^{5}$ Department of Transplantation Medicine, New York Presbyterian Hospital - Weill Cornell Medical Center, New York, NY, USA. ${ }^{6}$ Infectious Disease Services, Department of Medicine, Memorial Sloan Kettering Cancer Center, New York, NY, USA. ${ }^{7}$ Department of Biomedical Engineering, Cornell University, Ithaca, NY, USA.

*email: jrl2002@med.cornell.edu
} 
rinary tract infection (UTI) is one of the most common bacterial infections around the world, affecting over 150 million people yearly ${ }^{1}$. In the United States, it leads to over 10 million ambulatory visits and the economic burden is estimated to be greater than 2 billion dollars per year ${ }^{2}$. Kidney transplant recipients are a unique population to study the pathogenesis of UTI as they are frequently diagnosed with bacteriuria (positive urine culture without clinical symptoms) and UTI (positive urine culture with clinical symptoms of dysuria, frequency, urgency, or fever $)^{3}$. Bacteriuria occurs in $40 \%$ of kidney transplant recipients within the first year after transplantation ${ }^{4}$ and UTI occurs in 50\% within the first three years after transplantation ${ }^{5}$. Asymptomatic bacteriuria alone can have significant consequences in this patient population, increasing the risk of pyelonephritis seven-fold in a study of 189 kidney transplant recipients ${ }^{6}$. In most cases, bacteriuria and UTIs are treated effectively with antibiotics, but in rare cases, they can lead to morbidity and mortality. Approximately $1-3 \%$ of UTIs are associated with bacteremia development ${ }^{7,8}$, and UTIs occurring greater than 6 months after transplantation have been associated with increased risk of death and graft loss in a retrospective study of over 20,000 kidney transplant recipients ${ }^{5}$.

The pathogenesis of UTI is complex. Contamination of the periurethral space by a gut uropathogen is considered the initial step, followed by colonization of the urethra and bladder ${ }^{9}$. Elegant studies illuminating the colonization steps have elucidated the role of pili and flagella in the successful propagation of uropathogens during the development of $\mathrm{UTI}^{9}$. Few studies, however, have investigated the initial phase of UTI development and the role that gut microbiota may have. In a pilot study of 26 kidney transplant recipients in which we performed serial gut microbiota profiling, we reported an association between gut Enterococcus abundance and Enterococcus UTIs ${ }^{10}$. In another recent case-control study of children with UTIs and without UTIs, the relative gut abundance of Escherichia coli was significantly higher in children with UTIs than children without UTIs ${ }^{11}$. Both of these studies assessed whether the gut microbiota is associated with UTIs at the time of UTI, but not whether the gut microbiota is a risk factor for development of bacteriuria and UTI.

The primary focus of this study is to investigate the association between the gut microbiota and the risk of developing bacteriuria or UTI. In a cohort of kidney transplant recipients at our transplant center, conventional urine cultures are performed at every clinic visit, allowing for a detailed record of bacteriuria in this cohort. We collect serial fecal specimens from 168 kidney transplant recipients within the first 3 months of transplantation and perform 16S rRNA gene deep sequencing on these specimens to evaluate the gut microbiota's association with future development of bacteriuria and UTI. In a subset of subjects with bacteriuria, we evaluate urine supernatant specimens via cell-free DNA sequencing and paired fecal specimens via shotgun metagenomic sequencing to assess the strain similarity, uropathogenic genes, and antibiotic resistance genes. We report that gut abundance of uropathogens is associated with future development of bacteriuria and UTI and that strain analysis establishes a close strain level alignment between species found in the gut and in the urine in the same subjects with bacteriuria.

\section{Results}

Characteristics of the kidney transplant cohort. One hundred sixty-eight kidney transplant recipients provided a total of 510 fecal specimens in the first 3 months after transplantation. Fecal specimens were collected at post-transplant week 1 , week 2 , week 4 , and week 12 and during episodes of diarrhea as well as during episodes of UTIs. Conventional urine cultures were performed at every routine clinic visit (twice weekly in the first month, weekly in the second month, every 2 weeks in the third month, and monthly up to 6 months), allowing for a comprehensive evaluation of bacteriuria in these subjects. 16S rRNA gene deep sequencing of the V4-V5 hypervariable region was performed on the 510 fecal specimens and the mean average \pm standard deviation (SD) high quality classified reads were 18,179 $\pm 11,033$.

Among the 168 kidney transplant recipients, 102 subjects developed bacteriuria ( $\geq 10,000$ colony-forming units $[\mathrm{cfu}] / \mathrm{mL}$ ) (Bacteriuria Group) and 66 subjects did not in the first 6 months after transplantation (No Bacteriuria Group) (Supplementary Fig. 1). Differences in clinical and transplant characteristics are found in Supplementary Table 1. Female gender and older age were significantly associated with the Bacteriuria Group $(P<$ 0.05). The five most abundant bacteria identified included: 36 subjects with Escherichia species (spp.) bacteriuria, 36 subjects with Enterococcus spp. bacteriuria, 20 subjects with Klebsiella spp., 44 subjects with Staphylococcus spp., and 19 subjects with Streptococcus spp. The full list of bacteriuria episodes and the organisms isolated are found in Supplementary Table 2.

Gut abundance and respective bacteriuria group. We evaluated the relative gut abundance of the five most abundant genera associated with bacteriuria (Escherichia, Enterococcus, Klebsiella, Staphylococcus, and Streptococcus) and its relationship to respective bacteriuria group within the first 3 months of transplantation (Fig. 1). The Escherichia relative gut abundance was significantly higher in the Escherichia Bacteriuria Group than in the No Escherichia Bacteriuria Group (Fig. 1a) $(P<0.001$, Wilcoxon rank sum test), and the Enterococcus relative gut abundance was significantly higher in the Enterococcus Bacteriuria Group than in the No Enterococcus Bacteriuria Group $(P<0.001$, Wilcoxon rank sum test) (Fig. 1b). The relative gut abundances of Klebsiella, Staphylococcus, and Streptococcus were not significantly higher in their respective Bacteriuria Group than in their respective No Bacteriuria Group $(P>0.05$, Wilcoxon rank sum test) (Fig. 1c-e).

We further evaluated the relative gut abundance of Escherichia and Enterococcus in the cohort, stratified by gender and by older age (age $\geq 65$ ). The relative gut abundance of Escherichia was not significantly different between male samples and female samples $(P=0.16$, Wilcoxon rank sum test, Supplementary Fig. 2a) or between older age and younger age $(P=0.86$, Wilcoxon rank sum test, Supplementary Fig. 2b). The relative gut abundance of Enterococcus was significantly higher in the female samples than in the male samples $(P=0.007$, Wilcoxon rank sum test, Supplementary Fig. 2c) and the relative gut abundance of Enterococcus was significantly higher in the older age samples than in the younger age samples $(P=0.02$, Wilcoxon rank sum test, Supplementary Fig. 2d).

Gut abundance and the risk for bacteriuria and UTI. In order to account for the timing of the relative gut abundance with relationship to bacteriuria development, we utilized a timedependent Cox regression analysis to evaluate whether relative gut abundance of bacteria is a risk factor for future development of Escherichia and Enterococcus bacteriuria as these two genera were significantly different in the gut abundance analyses. To account for other clinical variables associated with bacteriuria, we analyzed clinical characteristics that we have previously analyzed ${ }^{7}$ as well as the cause of end stage renal disease and calculated panel reactive antibody status in multivariable Cox regression analysis. We utilized a cutoff of $1 \%$ relative abundance as this was the 

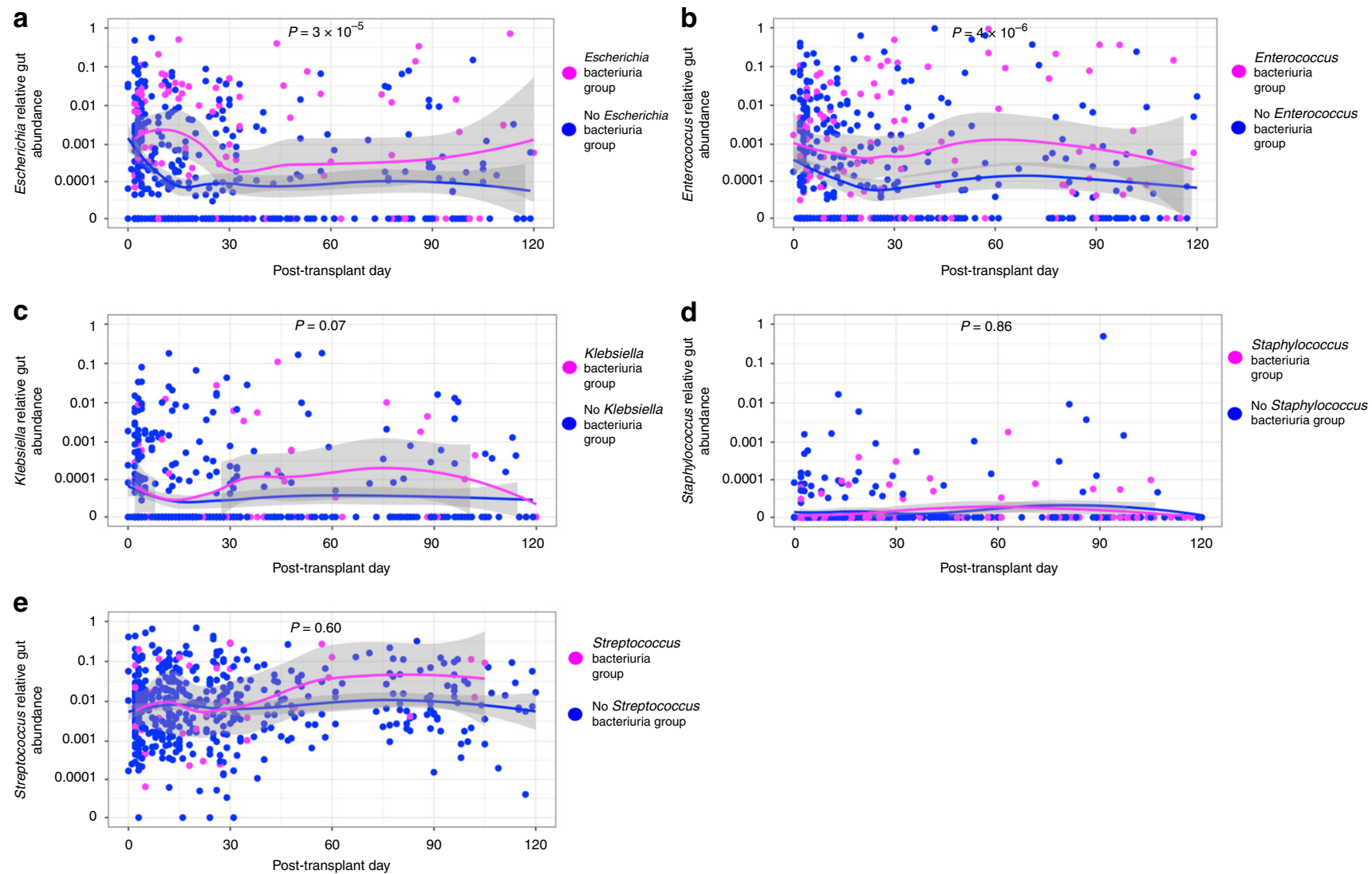

Fig. 1 Temporal dynamics of relative gut abundances and respective bacteriuria. The relative gut abundance of bacteria is on the $y$-axis (logarithmic scale) and the post-transplant days stool specimens were collected are on the $x$-axis. The 510 specimens are each represented by a magenta-colored point reflecting a specimen belonging to the respective Bacteriuria Group and a blue-colored point reflecting the respective No Bacteriuria Group. LOESS curves with $95 \%$ confidence intervals (in gray) were created by group status. Comparison of relative gut abundances by group status was performed using a Wilcoxon rank sum test. a Temporal dynamics of Escherichia relative gut abundance following kidney transplantation by Escherichia Bacteriuria Group status. b Temporal dynamics of Enterococcus relative gut abundance following kidney transplantation by Enterococcus Bacteriuria Group status. c Temporal dynamics of Klebsiella relative gut abundance following kidney transplantation by Klebsiella Bacteriuria Group status. d Temporal dynamics of Staphylococcus relative gut abundance following kidney transplantation by Staphylococcus Bacteriuria Group status. e Temporal dynamics of Streptococcus relative gut abundance following kidney transplantation by Streptococcus Bacteriuria Group status. Source data are provided as a source data file.

upper limit in the locally weighted scatterplot smoothing (LOESS) curves of Enterococcus and Escherichia graphs in Fig. 1.

Using a univariate Cox Regression analysis with the relative gut abundance as a time-dependent covariate, we found that a $1 \%$ relative gut abundance of Escherichia was associated with future development of Escherichia bacteriuria (Hazard Ratio [HR]: 2.8, $P=0.002$ ) (Table 1 ). In multivariate analysis that included the significantly associated univariate characteristics of female gender and cefazolin preoperative antibiotic prophylaxis, a $1 \%$ relative gut abundance of Escherichia continued to be associated with future development of Escherichia bacteriuria (HR: 2.8, $P=$ 0.002 ) (Table 1). We also found that a $1 \%$ relative gut abundance of Enterococcus was associated with future development of Enterococcus bacteriuria (HR: 2.4, $P=0.01$ ) (Table 2). In multivariate analysis that included the significantly associated univariate characteristics of deceased donor transplantation and delayed graft function, a $1 \%$ relative gut abundance of Enterococcus continued to be associated with future development of Enterococcus bacteriuria (HR: 2.3, $P=0.02$ ) (Table 2).

We also analyzed the relationship between $1 \%$ relative gut abundance of Escherichia and future development of Escherichia UTI. In univariate analysis, a $1 \%$ relative gut abundance of Escherichia was associated with future development of Escherichia UTI (HR: 2.9, $P=0.01$ ) and in multivariable analysis controlling for gender, a $1 \%$ relative gut abundance of Escherichia continue to be associated with future development of Escherichia UTI (HR: $2.8, P=0.02$ ) (Supplementary Table 3 ). In univariate analysis, we did not find a significant relationship between a $1 \%$ relative gut abundance of Enterococcus and future development of Enterococcus UTI (HR: 1.8, $P=0.28$ ) (Supplementary Table 4).

Strain similarity between gut and urine strains. To assess the relatedness of strains in the gut and in the urine, we evaluated 17 fecal specimens that were paired to 17 urine specimens in which urine cultures were positive for Escherichia coli $(n=14)$, Enterococcus faecalis $(n=2)$, and Enterococcus faecium $(n=2)$ (one urine culture was positive for both E. coli and E. faecalis) and in which relative gut abundance of Escherichia or Enterococcus was above two percent by $16 \mathrm{~S}$ rRNA gene sequencing. The timing of the collection of stool specimens in relationship to the collection of urine specimens is shown in Supplementary Fig. 3. The 2\% cutoff was chosen so that we would have at least an estimated $5 \mathrm{X}$ genome coverage of E. coli, E. faecalis, and E. faecium for strain analysis based on an estimated 40 million reads per sample and $50 \%$ alignment to human sequences.

The supernatants from these 17 urine specimens underwent single-stranded library preparation for cell-free DNA profiling ${ }^{12}$. Shotgun metagenomics sequencing was previously performed with an average mean $\pm S D$ depth of $55 \pm 8$ million reads ${ }^{13}$ with 
Table 1 Gut microbiota abundance and future development of Escherichia bacteriuria.

\begin{tabular}{|c|c|c|c|c|}
\hline \multirow[b]{2}{*}{ Characteristic } & \multicolumn{2}{|c|}{ Univariate analysis } & \multicolumn{2}{|c|}{ Multivariate analysis } \\
\hline & HR $(95 \% \mathrm{CI})$ & $P$ value & HR $(95 \% \mathrm{CI})$ & $P$ value \\
\hline Age, Years & $1.0(1.0-1.0)$ & 0.80 & & \\
\hline Female gender & $7.4(3.1-17.9)$ & 7.4E-06 & $7.9(3.3-18.9)$ & $4.2 E-06$ \\
\hline African American Race & $1.1(0.5-2.3)$ & 0.78 & & \\
\hline Diabetes mellitus & $1.2(0.6-2.4)$ & 0.58 & & \\
\hline Prior kidney transplant & $0.9(0.3-2.2)$ & 0.75 & & \\
\hline Cause of ESRD - DM & $1.1(0.6-2.3)$ & 0.71 & & \\
\hline Cause of ESRD - HTN & $0.6(0.2-1.8)$ & 0.40 & & \\
\hline$P R A \geq 80 \%$ & $1.3(0.5-3.7)$ & 0.60 & & \\
\hline Deceased donor transplantation & $1.4(0.7-2.8)$ & 0.32 & & \\
\hline Delayed graft function & $1.0(0.4-2.5)$ & 0.95 & & \\
\hline Cefazolin preoperative abx & $0.5(0.2-1.0)$ & 0.06 & $0.5(0.2-1.0)$ & 0.06 \\
\hline TMP-SMX PCP prophylaxis & $2.1(0.3-15.7)$ & 0.45 & & \\
\hline Anti-thymocyte globulin induction & $1.7(0.7-4.2)$ & 0.22 & & \\
\hline Prednisone maintenance & $1.1(0.6-2.3)$ & 0.71 & & \\
\hline 1\% Escherichia relative abundance & $2.8(1.4-5.4)$ & 0.002 & $2.8(1.5-5.5)$ & 0.002 \\
\hline
\end{tabular}

Table 2 Gut microbiota abundance and future development of Enterococcus bacteriuria.

\begin{tabular}{|c|c|c|c|c|}
\hline \multirow[b]{2}{*}{ Characteristic } & \multicolumn{2}{|c|}{ Univariate analysis } & \multicolumn{2}{|c|}{ Multivariate analysis } \\
\hline & HR $(95 \% \mathrm{Cl})$ & P value & HR $(95 \% \mathrm{Cl})$ & P value \\
\hline Age, Years & $1.0(1.0-1.0)$ & 0.31 & & \\
\hline Female gender & $1.7(0.9-3.2)$ & 0.13 & & \\
\hline African American Race & $1.2(0.6-2.5)$ & 0.65 & & \\
\hline Diabetes mellitus & $1.4(0.7-2.8)$ & 0.29 & & \\
\hline Prior kidney transplant & $1.0(0.4-2.5)$ & 0.97 & & \\
\hline Cause of ESRD-DM & $1.7(0.8-3.5)$ & 0.16 & & \\
\hline Cause of ESRD-HTN & $1.7(0.7-4.1)$ & 0.25 & & \\
\hline $\mathrm{PRA} \geq 80 \%$ & $1.6(0.6-4.6)$ & 0.36 & & \\
\hline Deceased donor transplantation & $2.2(1.1-4.2)$ & 0.02 & $1.8(0.8-4.1)$ & 0.17 \\
\hline Delayed graft function & $2.2(1.1-4.6)$ & 0.03 & $1.4(0.6-3.5)$ & 0.46 \\
\hline Cefazolin Preoperative Abx & $1.3(0.5-3.4)$ & 0.54 & & \\
\hline TMP-SMX PCP Prophylaxis & $1.0(0.2-4.2)$ & 0.99 & & \\
\hline Anti-thymocyte globulin induction & $0.8(0.4-1.6)$ & 0.51 & & \\
\hline Prednisone maintenance & $1.1(0.5-2.3)$ & 0.74 & & \\
\hline $1 \%$ Enterococcus relative abundance & $2.4(1.2-4.8)$ & 0.01 & $2.3(1.2-4.6)$ & 0.02 \\
\hline
\end{tabular}

A Cox Proportion Hazard Model was used to assess the relationship between gut microbial abundance and future development of Enterococcus bacteriuria. A $1 \%$ relative gut abundance of Enterococcus was assessed as a time-dependent covariate. The hazard ratio (HR) with $95 \%$ confidence intervals $(\mathrm{CI})$ is reported with the associated $P$ value. Univariate analysis was performed with all of the characteristics. Characteristics that were significantly associated with Enterococcus bacteriuria $(P<0.10)$ were further analyzed in the multivariate analysis. In bold text are the characteristics associated with future development of Enterococcus bacteriuria. Cause of ESRD is ordinal data and the reference for the HR is Cause of ESRD-Other. Source data are provided as a source data file DM diabetes mellitus, HR hazard ratio, HTN hypertension, PRA panel reactive antibodies, TMP-SMX trimethoprim-sulfamethoxazole, PCP Pneumocystis jiroveci

$75 \% \pm 12 \%$ (mean \pm SD) of sequences aligned to human chromosomes and removed. The 17 paired fecal specimens underwent shotgun metagenomic sequencing with an average mean $\pm \mathrm{SD}$ depth of $48 \pm 6$ million reads with $0.4 \% \pm 0.9 \%$ (mean \pm SD) sequences aligned to the human chromosomes and removed. Further details about the amount of urine DNA recovered and the estimated coverage of the E. coli, E. faecalis, and E. faecium genomes are found in the Supplementary Note 1.

We utilized StrainPhlAn ${ }^{14}$ to assess the relatedness of the strains in the urine and in the stool. In brief, StrainPhlAn maps sequences from a specimen against the MetaPhlAn2 marker database ${ }^{15}$ to produce a consensus-marker sequence for species in the sample. StrainPhlAn then utilizes MUSCLE ${ }^{16}$ to align consensus sequences from samples to produce a multiple sequence alignment and utilizes $\mathrm{RAxMl}^{17}$ to build the phylogenetic tree. Importantly, post-processing operations are utilized at each step to ensure high quality consensus sequences. In order to calculate differences at the base pair level, we utilized distmat from the European Molecular Biology Open Software Suite $^{18}$ which measures the number of single nucleotide variants (SNVs) per 100 base pairs.

Utilizing StrainPhlAn, we obtained 20 consensus strains of $E$. coli among all of the 34 urine and fecal specimens among which there were 6 pairs of $E$. coli urine and stool from the same subject. Figure $2 \mathrm{a}$ shows the related phylogeny of the $E$. coli consensus strains; 5 of the $6 E$. coli urine strains were closely related to the $E$. coli strains from the fecal specimens from the same subject. Using the SNVs per 100 base pair measurement, the closest strains for Subject 54 Urine 2, Subject 36 Urine, Subject 41 Urine, Subject 106 Urine, and Subject 165 Urine were the strains from the subjects' 
a

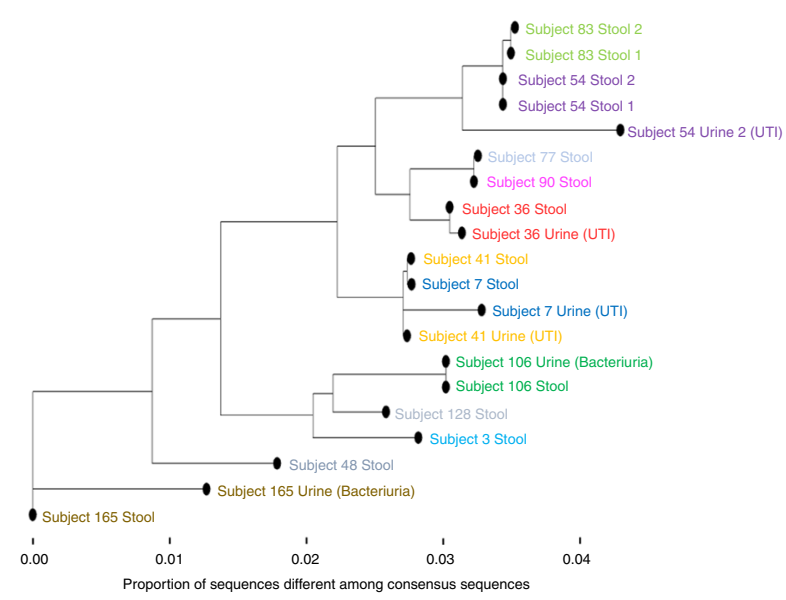

d

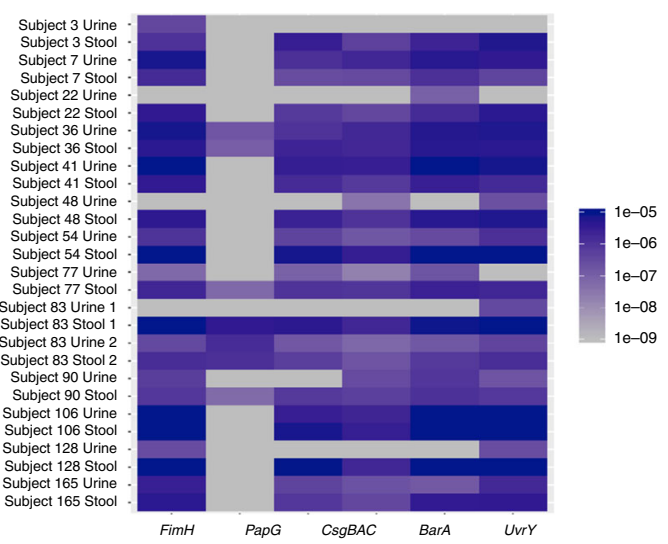

b

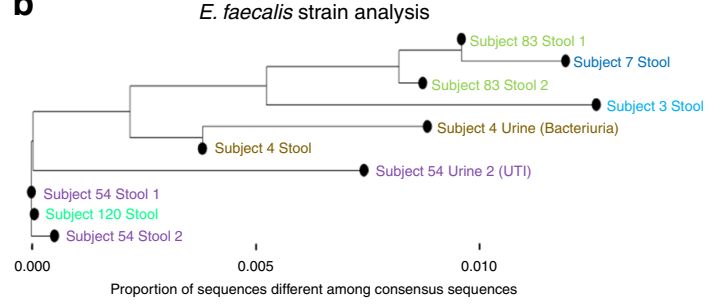

C

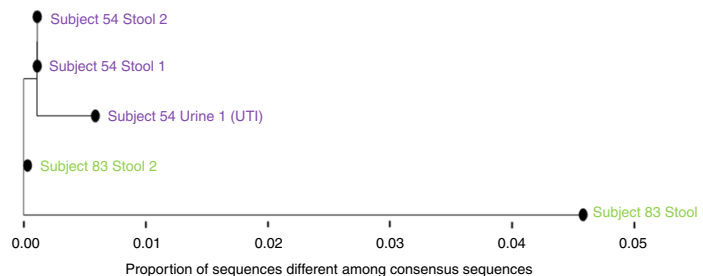

e

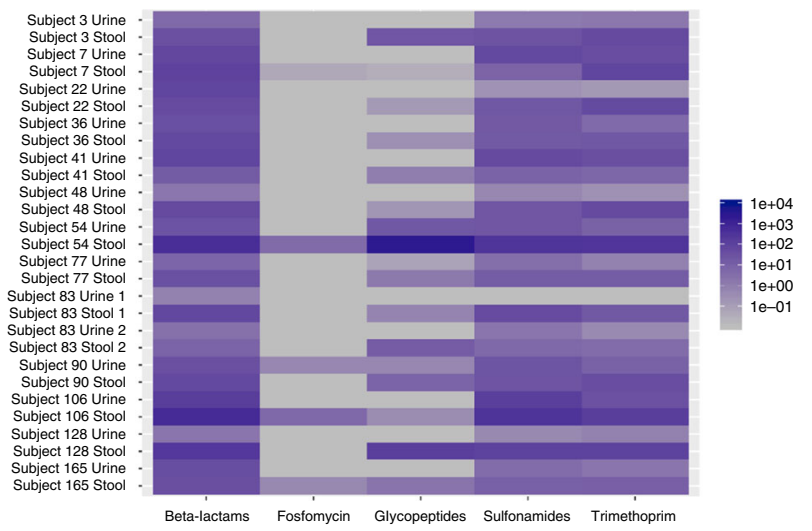

Fig. 2 Strain analysis, uropathogenic genes, and antimicrobial resistance genes in paired urine-fecal specimens. a Among 34 urine and fecal specimens profiled, 20 consensus strains for $E$. coli could be constructed using StrainPhIAn. A phylogenetic tree was constructed based on the E. coli strain alignment (24 markers) and the proportion of sequences that are different between strains is noted on the $x$-axis. Each point represents an $E$. coli strain from a urine or fecal specimen with different colors representing different subjects. b Among 34 urine and fecal specimens profiled, 10 consensus strains for $E$. faecalis could be constructed using StrainPhIAn. A phylogenetic tree was constructed based on the E. faecalis alignment (200 markers) and the proportion of sequences that are different between strains is noted on the $x$-axis. Each point represents an $E$. faecalis strain from a urine or fecal specimen with different colors representing different subjects. c Among 34 urine and fecal specimens profiled, 5 consensus strains for $E$. faecium could be constructed using StrainPhIAn. A phylogenetic tree was constructed based on the E. faecium alignment (200 markers) and the proportion of sequences that are different between strains is noted on the $x$-axis. Each point represents an $E$. faecium strain from a urine or fecal specimen with different colors representing different subjects. d Bacterial genes were determined using HUMAnN2 ${ }^{19}$ and relative abundance was estimated for each of the following uropathogenic E. coli associated genes: FimH, PapG, CsgBAC, BarA, and UvrY. A heatmap was constructed with the uropathogenic genes on the $X$-axis and the E. coli associated urine specimens and paired stool specimens on the $Y$-axis. The abundance is colored by blue intensity, log scaled. e Antibiotic resistance genes were determined using Bowtie $2^{21}$ on the MEGARES antibiotic resistance database ${ }^{22}$ and RPKM was estimated for genes that confer resistance to beta-lactams, fosfomycin, glycopeptides, sulfonamides, and trimethoprim. A heatmap was constructed with antibiotic resistance gene classes on the $X$-axis and the $E$. coli associated urine specimens and paired stool specimens on the $Y$-axis. The abundance is colored by blue intensity, log scaled. Source data are provided as a source data file.

own stool samples. The closest strain for Subject 7 Urine was the strain from Subject 41 Urine but very closely similar to the strain from Subject 7 Stool (Supplementary Table 5).

We obtained 10 consensus strains of $E$. faecalis among all of the 34 urine and fecal specimens among which there were 2 pairs of E. faecalis urine and stool from the same subject. Figure $2 \mathrm{~b}$ shows the related phylogeny of the E. faecalis consensus strains and that both E. faecalis urine strains were closely related to the E. faecalis strains from the fecal specimens from the same subject. Using the SNVs per 100 base pair measurement, the closest strains for Subject 4 Urine and Subject 54 Urine 2 were the strains from the subjects' own stool samples (Supplementary Table 6).

We obtained five consensus strains of E. faecium among all of the 34 urine and fecal specimens among which there was 1 pair of
E. faecium urine and stool from the same subject. Figure $2 \mathrm{c}$ shows the related phylogeny of the E. faecium consensus strains and that the E. faecium urine strain was closely related to the E. faecium strain from the fecal specimen from the same subject. Using the SNVs per 100 base pair measurement, the closest strains for Subject 54 Urine 1 was the strain from the subject's own stool sample (Supplementary Table 7).

Uropathogenic genes and antibiotic resistance genes. We further analyzed the 14 paired urine and fecal specimens associated with $E$. coli bacteriuria for bacterial genes. Bacterial gene alignments were determined using HUMAnN2 ${ }^{19}$ and normalized to relative abundance using total sum scaling. We evaluated the following uropathogenic genes associated with UTI: ${ }^{20} \mathrm{FimH}, \mathrm{PapG}, \mathrm{CsgBAC}$, 
BarA, and $U v r Y$. FimH is a gene that is part of the fim operon that encodes type 1 pili, allowing for attachment to the bladder epithelium; PapG is a gene that is part of the pap operon that encodes the P-type pilus, allowing for attachment to the bladder epithelium; $C s g B A C$ is part of the operons that coordinates curli formation, which can facilitate biofilm formation; BarA and $U v r Y$ are part of the two component signaling systems, which regulate metabolic pathways and allow for adaptation in the urinary tract ${ }^{20}$. We found the presence of these genes in both paired urine and fecal specimens (Fig. 2d). Specifically, FimH, CsgBAC, BarA, and $U v r Y$ were found in both the urine and fecal specimens from the same subject in a majority of the 14 paired urine and fecal specimens, supporting the similarity of urine and gut E. coli strains from the same subjects. Five of the 14 urine samples were associated with symptoms and there were no significant differences in the abundance of any of these genes between the symptomatic $E$. coli UTI and asymptomatic E. coli bacteriuria $(P>0.05$, Wilcoxon rank sum test), but there was a trend towards a higher abundance of $C s g B A C$ and BarA in the symptomatic $E$. coli UTI than the asymptomatic $E$. coli bacteriuria ( $P=0.08, \mathrm{P}=0.08$, Wilcoxon rank sum test, respectively) (Supplementary Fig. $4 \mathrm{a}-\mathrm{e})$.

We also analyzed the paired urine and fecal specimens for antibiotic resistance genes. Antibiotic resistance genes were determined using Bowtie $2^{21}$ on the MEGARES antibiotic resistance genes database $\mathrm{e}^{22}$ and were normalized to reads per kilobyte per million (RPKM). We evaluated genes conferring resistance to the following classes of antibiotics: beta-lactams, fosfomycin, glycopeptides, sulfonamides, and trimethoprim. Evaluation using the MEGARES antibiotic resistant genes database ${ }^{22}$ included 115 gene/operons in the beta-lactams class; 7 gene/operons in the fosfomycin class; 72 gene/operons in the glycopeptide class; 4 gene/ operons in the sulfonamides class; and 11 genes/operons in the trimethoprim class (The full list of genes/operons is provided in Supplementary Data 1.) Beta-lactams, sulfonamides, and trimethoprim resistance genes were present in both urine and fecal specimens from the same subject in a majority of the 14 paired fecal and urine specimens, supporting the similarity of urine and gut $E$. coli strains from the same subjects (Fig. 2e). In the 14 urine samples, we then evaluated for the presence of common and clinically significant classes of beta-lactamase genes: CTX-M, TEM, SHV, CMY, DHA, and OXA and correlated these results to antimicrobial susceptibility testing results from the uropathogen isolated in culture (Supplementary Fig. 5). We detected TEM genes, which confer ampicillin resistance, in 12 of the 13 urine samples that yielded ampicillin-resistant E. coli in culture. Furthermore, we detected CTX-M genes, which confer ampicillin and ceftriaxone resistance in all three urine specimens that yielded ampicillin- and ceftriaxone resistant $E$. coli in culture.

Antibiotics and gut Escherichia and Enterococcus abundance. We evaluated whether the most common antibiotics used in the cohort were risk factors for development of $1 \%$ relative gut abundance of Escherichia or $1 \%$ relative gut abundance of Enterococcus with the class of antibiotic administered as a timedependent covariate in Cox regression analysis. The list of antibiotics given in the cohort is found in Supplementary Table 8. Beta-lactams had an increased hazard ratio for development of $1 \%$ relative Enterococcus gut abundance (HR: 4.6, $P<0.001$ ) and cefazolin preoperative prophylaxis had a decreased hazard ratio for development of 1\% relative Escherichia gut abundance (HR: $0.4, P=0.03$ ) (Supplementary Table 9).

\section{Discussion}

The current study supports the notion that uropathogenic bacteria originate from the gut and further highlights a novel relationship between the gut microbiota and future development of bacteriuria and UTI in kidney transplant recipients. Prior studies have investigated the pathogenesis of UTI by assessing the mechanisms for survival and propagation of uropathogens in the urinary tract ${ }^{9}$. However, few studies have investigated the step preceding development of bacteriuria/UTI, the seeding of the urinary tract. In a pilot study, we evaluated the serial microbiome profiles in 26 kidney transplant recipients and reported that gut Enterococcus abundance was associated with Enterococcus UTIs ${ }^{10}$, but we were not able to assess whether gut Enterococcus abundance was a risk factor for future development of Enterococcus bacteriuria or UTI.

In this study, we found that the gut abundances of Escherichia and Enterococcus are associated with future development of Escherichia and Enterococcus bacteriuria, respectively, independent of clinical factors like gender. We further found that the gut abundance of Escherichia was associated with symptomatic Escherichia UTI development. We did not find a similar relationship between the gut abundance of Enterococcus and Enterococcus UTI, but it is possible that we did not have sufficient numbers since only 14 of the 36 Enterococcus bacteriuria episodes were classified as symptomatic Enterococcus UTI.

To further support the gut microbiota-UTI link, we demonstrate that the strains of E. coli and E. faecalis in the urine specimens are most similar to the strains of E. coli and E. faecalis in fecal specimens from the same subjects, respectively. It is customarily thought that uropathogens present in the gut are the source of UTI via periurethral contamination ${ }^{9}$. However, prior evidence to support this hypothesis is limited. In fact, a recent study found that women with $E$. coli UTIs had different E. coli strains in their urine and in their stool using pulsed-field gel electrophoresis ${ }^{23}$. Literature investigating the role of uropathogenic genes like FimH in increasing the propensity for gut E. coli strains to cause UTI is also relatively limited ${ }^{24}$. Recent studies have found increased gut abundance of Escherichia or Enterococcus in patients with UTI compared to patients without UTIs ${ }^{10,11}$, but they did not evaluate temporal dynamics of the gut microbiota. Our study expands upon this idea by demonstrating that increased fecal abundance of Escherichia and Enterococcus is associated with an increased risk of developing future bacteriuria with the respective organism. We also evaluated for the presence of uropathogenic genes like FimH and antibiotic resistance genes in paired E. coli urine and fecal specimens. We found the presence of these genes in both the urine and fecal specimens from the same subject in a majority of the samples, further supporting the strain alignment analysis that the E. coli in the gut is similar to the $E$. coli in the urine from the same subject. Interestingly, we did detect uropathogenic genes in asymptomatic E. coli bacteriuria cases. It is possible that the uropathogenic genes are present but not actively transcribed in these cases, that the presence of these gene products does not necessarily lead to symptomatic UTI, or that the cases were actually symptomatic but were not evaluated because the presence of urinary symptoms was not prospectively assessed.

Our study also investigated antibiotic usage associated with increased fecal Escherichia and Enterococcus abundances. We found that beta-lactams increased the risk for development of greater than 1\% relative gut abundance of Enterococcus. Enterococcus, particularly Enterococcus faecium, is inherently resistant to many antibiotics in this class, allowing expansion of Enterococcus in the setting of the depletion of other gut bacteria by these antibiotics. Our current data support the notion that antibiotics can alter the gut microbiota and increase abundances of pathogenic bacteria in the gut. Recent studies have reported that these dysbiotic states with pathogenic bacteria can lead to infectious complications beyond $C$. difficile infection. Taur et al. 
investigated the gut microbiome profiles of 94 bone marrow transplant recipients and reported that elevated gut Enterococcus abundances were associated with future development of Enterococcus bacteremia and that elevated gut Proteobacteria abundances were associated with future development of Proteobacteria bacteremia $^{25}$. In a study of 28 non-Hodgkin lymphoma patients, Montassier et al. reported that gut microbial diversity was a predictor of future development of bloodstream infections ${ }^{26}$.

The gut microbiota's relationship with bacteriuria development highlights a potential for novel gut microbiota-focused treatments. There are case reports that supports the notion that FMT could be a potential treatment for recurrent UTIs. In a case report of a heart and kidney transplant recipient who developed multiple events of Enterococcus bacteremia and UTIs as well as recurrent C. difficile infections, FMT decreased the fecal abundance of Enterococcus in the gut and led to resolution of Enterococcus infections in the subject for up to 14 months $^{27}$. In a case series of FMT for recurrent $C$. difficile infections in a non-transplant population, the number of UTIs in the year after FMT was significantly lower than the number of UTIs in the year prior to $\mathrm{FMT}^{28}$. In this case series, however, the fecal microbiome was not profiled prior to and after FMT.

An important consideration in this study is the potential for cross-contamination of urine and fecal specimens. However, we took several steps to minimize its impact. With respect to urine contamination of fecal specimens, we collected fecal specimens using the Fisherbrand Commode Specimen Collection kit which was used in the Human Microbiome Project ${ }^{29}$ and which, by design, minimizes cross-contamination of urine in fecal specimens $^{30}$. We also highlight the significant difference in bacterial biomass between fecal and urine specimens by several log folds ${ }^{31}$, making urine contamination of fecal specimens unlikely to affect our analyses. With respect to fecal contamination of urine specimens, subjects were asked to provide clean-catch midstream urine specimens as part of routine clinical care. We further highlight that our analyses also focused on evaluating the relationship between the gut microbiota and UTI. UTI is unlikely a contamination event because subjects experienced concomitant symptoms with the positive urine cultures and so this analysis is less likely to be affected by this type of contamination. Our study was also designed as a prospective collection of fecal specimens and fecal specimens were collected prior to the development of bacteriuria or UTI, making the collection processes independent of each other. Despite these steps taken to minimize cross-contamination, it is important to interpret the results of the study with due caution because cross-contamination of urine and fecal specimens is a potential confounder.

In addition, with respect to strain analysis, we did not have access to the urinary strains that were isolated for routine clinical care by the clinical microbiology laboratory. Unlike bloodstream isolates, urinary isolates are not saved for extended periods of time. However, we were able to utilize a computational algorithm (StrainPhlAn) to reconstruct consensus strains to evaluate the relatedness of the strains. While this approach provides an assessment of the genetic relatedness of the urine and fecal strains from the same subject, it is limited by the reconstruction of consensus strains from short base pair reads and may not reflect the full sequences of the strains that would be analyzable if the bacterial isolates were available. We also did not have urine from the donor to evaluate for the presence of donor-derived infections. However, of the 14 cases of E. coli bacteriuria for which strain analysis was performed, eight had a urine culture that was negative prior to the diagnosis of bacteriuria, suggesting the subjects developed the infection de novo. With respect to multivariable analysis, we included many variables that are associated with UTI in kidney transplant recipients, but we were not able to include acute rejection or prolonged foley catheter utilization because of the low number of events as well as presence of a ureteral stent since all subjects had a ureteral stent placed. We performed a retrospective chart review of symptoms to distinguish symptomatic UTI from asymptomatic bacteriuria; since this was not a prospective collection of symptoms, it is possible that we did not capture all cases of symptomatic UTIs. Finally, we were not able to assess for changes in gut microbiota prior to transplantation and after transplantation since we only had five subjects who provided specimens prior to transplantation.

Despite these limitations, our data support uropathogenic gut abundance as a risk factor for development of bacteriuria and UTI. Our results further support future studies on modulating the gut microbiota as a potential novel strategy for preventing UTIs, especially in cases of recurrent UTIs.

\section{Methods}

Kidney transplant cohort. Two-hundred eighty kidney transplant recipients who received kidney transplants during August 2015 to November 2016 were consented for serial collection of fecal and urine specimens, and 168 kidney transplant recipients provided at least 1 fecal specimen. The Weill Cornell Institutional Review Board approved this study and each subject gave written informed consent. No organs were procured from prisoners and all kidney transplant recipients were performed at NewYork-Presbyterian Hospital-Weill Cornell Medical Center. Urine specimens were analyzed at the clinical microbiology laboratory at NewYork-Presbyterian Hospital-Weill Cornell Medical Center. Each urine specimen was inoculated onto tryptic soy agar with sheep blood (Becton, Dickinson and Company [BD], Franklin Lakes, NJ) and MacConkey agar (BD) using a 1 microliter inoculation loop and incubated in ambient air at $35^{\circ} \mathrm{C}$. Bacteriuria was defined as having a positive urine culture $(\geq 10,000$ colony-forming units of a potential uropathogen per $\mathrm{mL}$ of urine) in the first 6 months after transplantation (up to 210 days post-transplantation). Clinical UTI was distinguished from bacteriuria by having bacteriuria and symptoms including: dysuria, frequency, urgency, or fevers ${ }^{3}$ in the first 6 months after transplantation; the symptoms were determined by chart review by a single individual. Bacterial isolates recovered in culture were identified and their antimicrobial susceptibility profiles were determined using standard identification and broth microdilution antimicrobial susceptibility testing platforms. Antimicrobial susceptibility data were interpreted using the Clinical and Laboratory Standards Institute M100 document depending on the year of the culture: M100-S25 32 (2015) or M100-S26 $6^{33}$ (2016). An a priori sample size determination was not performed given the limited preliminary data examining the relationship between gut microbial abundance and bacteriuria.

Fecal specimen collections. Subjects were given a Fisherbrand toilet specimen collection kit (Fisher Scientific, New Hampton, NH, USA) and were asked to provide a fecal specimen. As an inpatient, stool specimens were stored at $4{ }^{\circ} \mathrm{C}$ and as an outpatient, subjects stored the specimen with ice packs. Fecal specimens were collected and aliquoted into approximately $200 \mathrm{mg}$ aliquots and stored at $-80^{\circ} \mathrm{C}$ Fecal specimens were collected at post-transplant week 1 , week 2 , week 4 , and week 12 and during episodes of diarrhea as well as during episodes of UTIs. Analysis was restricted to the 510 fecal specimens within the first 3 months after transplantation (up to 120 days post-transplantation) except for two additional fecal specimens from kidney transplant recipients who had recurrent bacteriuria (These samples were used for strain analysis.) With respect to stool samples and routine urine samples being obtained at the same time, only 28 (5\%) were collected at the same time during the clinical visit. In most cases, stool samples were brought from home $(n=269,53 \%)$ or collected in the hospital $(n=213,42 \%)$.

DNA extraction, 16S rRNA gene amplification, and sequencing. DNA extrac tion, 16S rRNA gene amplification, and deep sequencing were performed ${ }^{34}$. The team performing DNA extraction, 16S rRNA gene amplification, and deep sequencing was blinded to the Bacteriuria Group status of the specimens. In brief, DNA was extracted from a frozen stool aliquot $(<500 \mathrm{mg})$ using a solution containing $500 \mu \mathrm{L}$ of extraction buffer $(200 \mathrm{mM}$ TrisHCl [pH 8.0]/200 mM NaCl/20 mM EDTA), $500 \mu \mathrm{L}$ of phenol:chloroform:isoamyl alcohol (25:24:1), $200 \mu \mathrm{L}$ of $20 \%$ SDS, and $500 \mu \mathrm{L}$ of $0.1-\mathrm{mm}$-diameter zirconia/silica beads (BioSpec Products, Bartlesville, OK, USA). Mechanical disruption of the stool specimen was performed using a bead beater for $2 \mathrm{~min}$, followed by two more rounds of phenol:chloroform isoamyl alcohol extraction. DNA was isolated with ethanol precipitation and was resuspended in $50 \mu \mathrm{L}$ of $1 \mathrm{X}$ TE buffer with $100 \mu \mathrm{g} / \mathrm{mL}$ RNase. The DNA was further purified using QIAamp mini spin columns (Qiagen, Hilden, Germany). For each sample, duplicate $50 \mu \mathrm{L}$ PCR reactions were performed, each containing $50 \mathrm{ng}$ of purified DNA, $0.2 \mathrm{mM}$ dNTPs, $1.5 \mathrm{mM} \mathrm{MgCl}_{2}, 2.5 \mathrm{U}$ Platinum Taq DNA polymerase, $2.5 \mu \mathrm{L}$ of $10 \times$ PCR buffer, and $0.5 \mu \mathrm{M}$ of each primer designed to amplify the $16 \mathrm{~S}$ rRNA gene V4-V5 region: $563 \mathrm{~F}$ (5'-nnnnnnnn-NNNNNNNNNNNN-A YTGGGYDTAAAGNG-3') and $926 \mathrm{R}$ (5'-nnnnnnnn-NNNNNNNNNNNN-CC 
GTCAATTYHTTTRAGT-3'). A unique 12-base Golay barcode (Ns) precedes primers for identification of the sample ${ }^{35}$, and one to eight additional nucleotides precedes the barcode to offset the sequencing of the primers. Cycling conditions were $94^{\circ} \mathrm{C}$ for $3 \mathrm{~min}$, followed by 27 cycles of $94^{\circ} \mathrm{C}$ for $50 \mathrm{~s}, 51^{\circ} \mathrm{C}$ for $30 \mathrm{~s}$, and $72{ }^{\circ} \mathrm{C}$ for $1 \mathrm{~min}$. The final elongation step was at $72{ }^{\circ} \mathrm{C}$ for $5 \mathrm{~min}$. Replicate PCRs were pooled, and the PCR products were further purified using the Qiaquick PCR Purification Kit (Qiagen, Hilden, Germany). PCR amplicons were quantified and pooled at equimolar amounts and were ligated with Illumina barcodes and adaptors using the Illumina TruSeq Sample Preparation protocol (Illumina Inc, San Diego, CA, USA). The completed library was sequenced on an Illumina Miseq platform with a paired end $250 \mathrm{bp} \times 250 \mathrm{bp}$ kit.

$16 \mathrm{~S}$ rRNA sequencing bioinformatics. The $16 \mathrm{~S}$ rRNA gene paired-end reads were merged and demultiplexed and run with the UPARSE ${ }^{36}$ pipeline to: (a) perform error filtering, using maximum expected error ${ }^{37}$, (b) group sequences into operational taxonomic units (OTUs) (97\% distance-based similarity), and (c) identify and remove potential chimeric sequences, using de novo and reference-based methods. Taxonomic assignment was performed by using a custom Python script incorporating nucleotide BLAST ${ }^{38}$, with NCBI RefSe ${ }^{39}$ as a reference training set. We utilized NCBI RefSeq as the training set as we have previously done ${ }^{34,40,41}$ because it can capture species-level resolution with curation and manual inspection of the results and submission sources. A minimum $E$-value threshold of $1 \times 10^{-10}$ for assignments was utilized.

Statistical analyses. The distribution of continuous variables was compared using the two-tailed Wilcoxon rank sum test; the distribution of categorical variables was compared using two-tailed Fisher's exact test. A Cox Regression Hazard Model was used to estimate whether a relative fecal abundance of a specific organism was associated with development of bacteriuria of the same organism with relative fecal abundance as a time-ever dependent covariate where it was assumed that the abundance value was not crossed until the first time the microbial abundance value crossed the threshold. A multivariable Cox regression hazard model was performed to evaluate clinical variables that we have previously analyzed ${ }^{7}$ as well as the cause of end stage renal disease and calculated panel reactive antibody status. A similar Cox regression hazard model was used to estimate whether antibiotic class was associated with development of a relative fecal abundance of Enterococcus or Escherichia as a time-dependent covariate. All analyses were performed in R 3.3.3 with packages yingtools2 0.0.0.89, phyloseq 1.19.1, dplyr 0.7.7, tibble 2.1.1, reshape2 1.4.3, data.table $1.10 .4-3$, scales 0.5 .0 , Hmisc 4.1-1, stringr 1.4.0, stringi 1.4.3, biomformat 1.2.0, deplyr 0.7.7, coxphf 1.12, and ggtree 1.11.6.

Strain analysis for paired urine and fecal specimens. Urine specimens were also obtained serially in the kidney transplant recipients as part of a biobank in our center. Seventeen urine specimens had urine cultures that were positive for $E$. coli $(n=14)$, E. faecalis $(n=2)$, and E. faecium $(n=2)$ (one sample was positive for both $E$. coli and E. faecalis). Fecal specimens closest to the urine specimen and also had a relative gut abundance of greater than 2\% of either Escherichia or Enterococcus were chosen as the paired specimens. The $2 \%$ cutoff was chosen so that we would have at least an estimated $5 \mathrm{X}$ genome coverage of E. coli, E. faecalis, and $E$. faecium for strain analysis.

The urine specimens were centrifuged at $2000 \times g$ for $30 \mathrm{~min}$ on the same day of collection and the urine supernatant was stored at $-80^{\circ} \mathrm{C}$ in $1 \mathrm{~mL}$ aliquots. Cellfree DNA was extracted from the urine using the Qiagen Circulating Nucleic Acid Kit (Qiagen). Extracted cell-free DNA amounts were quantified with a Qubit Fluorometer using the HS dsDNA kit (Qubit, London, United Kingdom). A singlestranded library preparation method was used ${ }^{13}$. In brief, cell-free DNA was denatured and was ligated to biotinylated oligonucleotides. Primer extension was performed on streptavidin functionalized magnetic beads. Another set of adapters was ligated and the product was amplified with PCR (4-12 cycles).

Characterization of the library was performed with the AATI fragment analyzer and samples were pooled and sequenced on an Illumina Next Seq Instrument (paired-end, $75 \mathrm{bp} \times 75 \mathrm{bp})^{13}$. Given potential low biomass, we included a control with template DNA (IDT-DNA synthetic oligo mix lengths $25,40,55,70 \mathrm{bp} ; 0.20$ $\mu \mathrm{M}$ eluted in TE buffer) in each sample batch.

The 17 stool samples underwent DNA extraction as described above and were quantified using a Qubit Fluorometer (Qubit). Sequencing libraries were prepared using an Illumina Tru Seq Kit (Illumina Inc, San Diego, CA, USA). Samples were pooled and sequenced on an Illumina Hi Seq 4000 instrument (paired-end, $100 \mathrm{bp} \times 100 \mathrm{bp}$ ).

We utilized kneaddata v.0.5.2 using bowtie2 $\mathrm{v} 0.1^{21}$ to trim paired fastq files and remove human sequences. Microbial sequence coverage was calculated by the reads after trimming and after decontamination of human sequences, multiplied by the fraction of the organism estimated by MetaPhlAn2 2.6.015, multiplied by the number of base pairs per read, and divided by the genome length of the organism. We then utilized StrainPhlAn 2.6.0 ${ }^{14}$ to obtain consensus strains for E. coli, E. faecalis, and E. faecium. MetaPhlAn2 $2.6 .0^{15}$ was utilized to analyze the concatenated fastq file after kneaddata; the sample2markers script was utilized to obtain marker information for each sample; and generation of trees was constructed from alignments with StrainPhlAn script. Marker information for $E$. coli, E. faecalis, and E. faecium was extracted from the MetaPhlAn2 database. The default relaxed 2 StrainPhlAn parameters were utilized (marker_in_clade 0.2, sample_in_marker 0.2, N_in_marker 0.8, gap_in_sample 0.8). The tree was further analyzed using a modified version of the StrainPhlAn ggtree R script.

Uropathogenic/antibiotic resistance gene classification. Concatenated fastq files after kneaddata were analyzed using HUMAnN2 ${ }^{19}$. All bacterial genes were normalized to relative abundance using total sum scaling. A heatmap was generated for the paired E. coli urine and stool samples.

Concatenated fastq files after kneaddata were aligned using Bowtie $2^{21}$ sensitive mode to a MEGARES antibiotic resistance gene database ${ }^{22}$. The antibiotic resistance genes were normalized to reads per kilobyte per million (RPKM) and were grouped into classes of antibiotics. A heatmap was generated for the paired $E$. coli urine and stool samples.

Reporting summary. Further information on research design is available in the Nature Research Reporting Summary linked to this article.

\section{Data availability}

All sequencing data as well as the deidentified clinical data that support the findings of this study have been uploaded to the database of Genotypes and Phenotypes ( $\mathrm{dbGaP}$ ) with accession number phs001879.v1.p1. Local institutional review board approval will be needed to access the sequencing data as well as the deidentified clinical data. The source data underlying Figs. 1, 2, Tables 1, 1, Supplementary Figs. 1-5, and Supplementary Tables 1-9 are provided as a Source Data file.

\section{Code availability}

Custom python script for taxonomic assignment and R script for graphing phylogenetic tree can be found in a zip file in Supplementary Information.

\section{Received: 19 March 2019; Accepted: 4 November 2019;} Published online: 04 December 2019

\section{References}

1. Stamm, W. E. \& Norrby, S. R. Urinary tract infections: disease panorama and challenges. J. Infect. Dis. 183, S1-S4 Suppl 1 (2001).

2. Foxman, B. Urinary tract infection syndromes: occurrence, recurrence, bacteriology, risk factors, and disease burden. Infect. Dis. Clin. North Am. 28, 1-13 (2014).

3. Hollyer, I. \& Ison, M. G. The challenge of urinary tract infections in renal transplant recipients. Transpl. Infect. Dis. 20, e12828 (2018).

4. Ariza-Heredia, E. J. et al. Impact of urinary tract infection on allograft function after kidney transplantation. Clin. Transpl. 28, 683-690 (2014).

5. Abbott, K. C. et al. Late urinary tract infection after renal transplantation in the United States. Am. J. Kidney Dis.: Off. J. Natl. Kidney Found. 44, 353-362 (2004).

6. Fiorante, S. et al. Systematic screening and treatment of asymptomatic bacteriuria in renal transplant recipients. Kidney Int. 78, 774-781 (2010).

7. Lee, J. R. et al. Independent risk factors for urinary tract infection and for subsequent bacteremia or acute cellular rejection: a single-center report of 1166 kidney allograft recipients. Transplantation 96, 732-738 (2013).

8. Yalci, A. et al. Evaluation of infectious complications in the first year after kidney transplantation. Transpl. Proc. 47, 1429-1432 (2015).

9. Flores-Mireles, A. L., Walker, J. N., Caparon, M. \& Hultgren, S. J. Urinary tract infections: epidemiology, mechanisms of infection and treatment options. Nat. Rev. Microbiol. 13, 269-284 (2015).

10. Lee, J. R. et al. Gut microbial community structure and complications after kidney transplantation: a pilot study. Transplantation 98, 697-705 (2014).

11. Paalanne, N. et al. Intestinal microbiome as a risk factor for urinary tract infections in children. Eur. J. Clin. Microbiol. Infect. Dis. 37, 1881-1891 (2018).

12. Burnham, P. et al. Single-stranded DNA library preparation uncovers the origin and diversity of ultrashort cell-free DNA in plasma. Sci. Rep. 6, 27859 (2016).

13. Burnham, P. et al. Urinary cell-free DNA is a versatile analyte for monitoring infections of the urinary tract. Nat. Commun. 9, 2412 (2018).

14. Truong, D. T., Tett, A., Pasolli, E., Huttenhower, C. \& Segata, N. Microbial strain-level population structure and genetic diversity from metagenomes. Genome Res. 27, 626-638 (2017).

15. Truong, D. T. et al. MetaPhlAn2 for enhanced metagenomic taxonomic profiling. Nat. Methods 12, 902-903 (2015).

16. Edgar, R. C. MUSCLE: multiple sequence alignment with high accuracy and high throughput. Nucleic Acids Res. 32, 1792-1797 (2004). 
17. Stamatakis, A. RAxML version 8: a tool for phylogenetic analysis and post-analysis of large phylogenies. Bioinformatics 30, 1312-1313 (2014).

18. Rice, P., Longden, I. \& Bleasby, A. EMBOSS: the European Molecular Biology Open Software Suite. Trends Genet. 16, 276-277 (2000).

19. Franzosa, E. A. et al. Species-level functional profiling of metagenomes and metatranscriptomes. Nat. Methods 15, 962-968 (2018).

20. Terlizzi, M. E., Gribaudo, G. \& Maffei, M. E. UroPathogenic Escherichia coli (UPEC) infections: virulence factors, bladder responses, antibiotic, and nonantibiotic antimicrobial strategies. Front. Microbiol. 8, 1566 (2017).

21. Langmead, B. \& Salzberg, S. L. Fast gapped-read alignment with Bowtie 2. Nat. Methods 9, 357-359 (2012).

22. Lakin, S. M. et al. MEGARes: an antimicrobial resistance database for high throughput sequencing. Nucleic Acids Res. 45, D574-D580 (2017).

23. Bahadori, M. et al. Genetic relatedness of the Escherichia coli fecal population and strains causing urinary tract infection in the same host. Microbiologyopen 8, e00759 (2018)

24. Hashemizadeh, Z., Kalantar-Neyestanaki, D. \& Mansouri, S. Association between virulence profile, biofilm formation and phylogenetic groups of Escherichia coli causing urinary tract infection and the commensal gut microbiota: a comparative analysis. Micro. Pathog. 110, 540-545 (2017).

25. Taur, Y. et al. Intestinal domination and the risk of bacteremia in patients undergoing allogeneic hematopoietic stem cell transplantation. Clin. Infect. Dis. 55, 905-914 (2012).

26. Montassier, E. et al. Pretreatment gut microbiome predicts chemotherapyrelated bloodstream infection. Genome Med. 8, 49 (2016).

27. Stripling, J. et al. Loss of vancomycin-resistant enterococcus fecal dominance in an organ transplant patient with clostridium difficile colitis after fecal microbiota transplant. Open Forum Infect. Dis. 2, ofv078 (2015).

28. Tariq, R. et al. Fecal microbiota transplantation for recurrent Clostridium difficile infection reduces recurrent urinary tract infection frequency. Clin. Infect. Dis. 65, 1745-1747 (2017).

29. Human Microbiome Project Consortium. Structure, function and diversity of the healthy human microbiome. Nature 486, 207-214 (2012).

30. $\mathrm{Wu}, \mathrm{W}$. K. et al. Optimization of fecal sample processing for microbiome study-the journey from bathroom to bench. J. Formos. Med. Assoc. 118, 545-555 (2019).

31. Karstens, L. et al. Community profiling of the urinary microbiota: considerations for low-biomass samples. Nat. Rev. Urol. 15, 735-749 (2018).

32. CLSI. M100-S25. Performance Standards for Antimicrobial Susceptibility Testing; 25th Informational Supplement (CLSI, Wayne, PA, 2015).

33. CLSI. M100-S26. Performance Standards for Antmicrobial Susceptibility Testing: 26th Informational Supplement (CLSI, Wayne, PA, 2016).

34. Lee, J. R. et al. Gut microbiota dysbiosis and diarrhea in kidney transplant recipients. Am. J. Transplant 19, 488-500 (2018).

35. Caporaso, J. G. et al. Ultra-high-throughput microbial community analysis on the Illumina HiSeq and MiSeq platforms. ISME J. 6, 1621-1624 (2012).

36. Edgar, R. C. UPARSE: highly accurate OTU sequences from microbial amplicon reads. Nat. Methods 10, 996-998 (2013).

37. Edgar, R. C. \& Flyvbjerg, H. Error filtering, pair assembly and error correction for next-generation sequencing reads. Bioinformatics 31, 3476-3482 (2015).

38. Altschul, S. F., Gish, W., Miller, W., Myers, E. W. \& Lipman, D. J. Basic local alignment search tool. J. Mol. Biol. 215, 403-410 (1990).

39. Tatusova, T., Ciufo, S., Fedorov, B., O’Neill, K. \& Tolstoy, I. RefSeq microbial genomes database: new representation and annotation strategy. Nucleic Acids Res. 42, D553-D559 (2014).

40. Haak, B. W. et al. Impact of gut colonization with butyrate-producing microbiota on respiratory viral infection following allo-HCT. Blood 131, 2978-2986 (2018).

41. Taur, Y. et al. Reconstitution of the gut microbiota of antibiotic-treated patients by autologous fecal microbiota transplant. Sci. Transl. Med. 10, eaap9489 (2018).

\section{Acknowledgements}

This research work was supported, in part, by the National Institutes of Health grants: K23AI124464 (J.R.L.), R21AI133331 (J.R.L., I.D.V.), and R37AI051652 (M.S.); by a Chinese American Medical Society Summer Research Fellowship (L.Z.); and by a Summer Research Fellowship from the Weill Cornell Clinical and Translational Science Center (N.A.S.)

\section{Author contributions}

M.M. was involved in the conception of the study, in the 16S rRNA deep sequencing of fecal samples, in data analysis, and in writing of the manuscript. N.A.S., C.G., L.Z., E.E., J. H., and S.A. were involved in data collection and analysis and in writing of the manuscript. M.J.S., L.F.W., C.C., D.M.D., M.L., Y.T., and E.L. were involved in data analysis and in writing of the manuscript. L.L. was involved in the 16S rRNA gene deep sequencing of fecal samples. P.B. and I.D.V. were involved in the cell-free DNA sequencing of urine samples, data analysis, and in writing of the manuscript. E.P. and $M$ S. were involved in the conception of the study, in data analysis, and in writing of the manuscript. J.R.L. was involved in the conception of the study, in data analysis and collection, and in writing of the manuscript.

\section{Competing interests}

The authors of this manuscript have the following competing interests to disclose. M.J.S. receives research support from Allergan, Merck, Contrafect, and BioFire Diagnostics, and received consulting fees from Acahogen, Inc. and Shionogi; L.F.W. receives research support from Accelerate Diagnostics, Inc and BioFire Diagnostics, LLC; C.C. receives support from Redhill, MERCK, Rebiotix, Seres, and Finch Therapeutics.; E.P. has received speaker honoraria from Bristol Myers Squibb, Celgene, Seres Therapeutics, MedImmune, Novartis and Ferring Pharmaceuticals and is an inventor on patent application \#WPO2015179437A1, entitled Methods and compositions for reducing Clostridium difficile infection and \#WO2017091753A1, entitled Methods and compositions for reducing vancomycin-resistant enterococci infection or colonization and holds patents that receive royalties from Seres Therapeutics, Inc.; J.R.L. receives research support from BioFire Diagnostics, LLC. D.D., P.B., I.D.V., M.S., and J.R.L. are inventors on patent application \#W02018187521A2 entitled Methods of detecting cell-free DNA in biological samples.

\section{Additional information}

Supplementary information is available for this paper at https://doi.org/10.1038/s41467019-13467-w.

Correspondence and requests for materials should be addressed to J.R.L.

Reprints and permission information is available at http://www.nature.com/reprints

Peer Review Information Nature Communications thanks Tessa Andermann, Tara Sigdel, and other, anonymous, reviewer(s) for their contribution to the peer review of this work. Peer reviewer reports are available.

Publisher's note Springer Nature remains neutral with regard to jurisdictional claims in published maps and institutional affiliations.

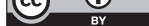

Open Access This article is licensed under a Creative Commons Attribution 4.0 International License, which permits use, sharing, adaptation, distribution and reproduction in any medium or format, as long as you give appropriate credit to the original author(s) and the source, provide a link to the Creative Commons license, and indicate if changes were made. The images or other third party material in this article are included in the article's Creative Commons license, unless indicated otherwise in a credit line to the material. If material is not included in the article's Creative Commons license and your intended use is not permitted by statutory regulation or exceeds the permitted use, you will need to obtain permission directly from the copyright holder. To view a copy of this license, visit http://creativecommons.org/ licenses/by/4.0/.

(C) The Author(s) 2019 